\title{
A chemical approach to stem cell biology
}

\author{
Sheng Ding ${ }^{1}$ \\ ${ }^{1}$ The Scripps Research Institute, USA
}

Recent advances in stem cell biology may make possible new approaches for the treatment of a number of diseases. For better understanding of the molecular mechanisms that control stem cell fates as well as an improved ability to manipulate these cells, we have developed and implemented chemical and functional genomic tools, including high throughput cell-based phenotypic screens of arrayed chemical, cDNA and RNAi libraries, and performed genomic and proteomic profiling of homogenous undifferentiated/self-renewing or selectively differentiated cell populations under chemically defined conditions. In-depth biochemical and functional assays in vitro and in vivo, have been applied to identify and further characterize small molecules and genes that can control stem cell fate in various systems.

Keywords: pluripotency, reprogramming, high throughput screening, chemical biology

Cell Research (2008) 18:s5. doi: 10.1038/cr.2008.95; published online 4 August 2008

Correspondence: Sheng Ding

E-mail: sding@scripps.edu

Sheng Ding obtained his BS with honors from Caltech in 1999, and his $\mathrm{PhD}$ from The Scripps Research Institute in 2003. He then stayed and joined the faculty of the Chemistry Department at Scripps as an Assistant Professor, and then Associate Professor in 2007. The main research focus of the Ding laboratory is to develop and integrate chemical and functional genomic tools to study stem cell biology and regeneration. Ding lab has developed and implemented high throughput cellular screens of these libraries to identify small molecules and genes which can control stem cell fate in various systems including (i). Selfrenewal, as well as directed neuronal, cardiac and pancreatic differentiations of pluripotent mouse and human embryonic stem cells; (ii). Directed neuronal differentiation and subtype neuron specification of human and rodent neural stem cells; (iii). Directed differentiation of mesenchymal stem cells to osteogenic, adipogenic, chondrogenic and myogenic lineages; (iv). Functional proliferation of adult cardiomyocytes and islets/beta cells; (v). Cellular plasticity and dedifferentiation of lineagerestricted somatic cells; (vi). Developmental signaling pathways. Moreover, systemic biochemical and cellular studies, including detailed structure-activity-relationship (SAR) studies, affinity chromatography for target identification, genomewide expression analysis using Affymetrix microarrays, and cDNA and/or RNAi complementation screens to map signaling pathways, are being used to characterize the molecular mechanism of these identified small molecules and genes. Those studies may ultimately facilitate the therapeutic application of stem cells and the development of small molecule drugs to stimulate tissue and organ regeneration in vivo. 